\title{
Nanomedicine hitchhikes on neutrophils to the inflamed lung
}

\author{
Supramolecular arrangement of proteins provides nanoparticles with neutrophil tropism via complement \\ opsonization during an acute inflammation, enabling diagnosis and treatment of acute lung injury.
}

\author{
Jeonghwan Kim and Gaurav Sahay
}

$\Lambda$ cute lung injury (ALI), and its more fatal form, acute respiratory distress syndrome (ARDS), are life-threatening forms of respiratory failure with high morbidity and mortality rates ${ }^{1}$. ALI/ARDS can be caused by pneumonia, aspiration of gastric contents, sepsis and other etiologies. ARDS is a frequent complication in severe acute respiratory syndrome coronavirus 2 (SARS-CoV-2) infection, and its incident is nearly $90 \%$ among non-survivors of coronavirus disease 2019 (COVID-19) ${ }^{2}$. Despite the severity of ARDS, there is no effective pharmacologic therapy ${ }^{3}$. Inhaled nitric oxide, glucocorticoids and $\beta$-agonists have been tested for ARDS treatment; however, they either fail to reduce mortality, show only transient improvements in symptoms or result in adverse effects in other organs. So far, mechanical ventilation is the first-line treatment that enhances survival in patients with ARDS.

Neutrophils account for the largest portion of circulating leukocytes and are frontline responders of the innate immune system during an acute inflammation. In inflamed lungs, lung-resident cells (for example, macrophages) secrete various chemokines, such as CXCL8 (interleukin $8)$, CCL2 and CCL7, that prime and recruit neutrophils and monocytes to the lungs ${ }^{3,4}$. Infiltration of neutrophils into the extravascular compartments of lungs is a defining sign of ALI/ARDS ${ }^{3}$. Activated neutrophils release neutrophil extracellular traps, oxidants and proteases, which aggravate lung injury. Such toxic mediators damage the lung epithelial and endothelial membranes, resulting in the influx of protein-rich edema to alveolar and interstitial spaces and arterial hypoxemia. Therefore, ideal strategies to treat ALI/ ARDS would limit the activation and recruitment of neutrophils while preventing edema and loss of functions in the lungs.

Nanoparticles are a versatile tool in medicine, with roles in gene therapy and immunotherapy, and can be engineered to interact with target cells in a selective manner. Previous attempts to direct nanoparticles to neutrophils have explored conjugating antibodies ${ }^{5}$, altering particle compositions ${ }^{6}$ or fine-tuning physicochemical properties ${ }^{7}$. Writing in Nature Nanotechnology, Myerson and colleagues report novel design criteria to induce selective uptake of nanoparticles by neutrophils marginated to inflamed lungs $^{8}$ (Fig. 1). In vivo screening of a library consisting of 23 nanoparticle formulations reveals that selected nanoparticles preferentially accumulate in the lungs in the ALI/ARDS animal model. This pulmonary accumulation of nanoparticles is driven by the presence on their surface of 'agglutinated' proteins, supramolecular arrangements of proteins, resulting from hydrophobic interactions, -specific crosslinking or electrostatic interactions of proteins. The authors term these nanoparticles 'nanoparticles with agglutinated proteins (NAPs) ${ }^{3}$. NAPs show a propensity to accumulate in the inflamed lungs, independent of their size, zeta-potential and protein composition. This characteristic biodistribution results from preferential uptake of the nanoparticles in neutrophils migrating to the lungs in response to inflammation. In contrast, nanostructures with symmetric or regular arrangements (for example, viral capsids and ferritin nanocages), non-protein nanoparticles (for example, liposomes and polystyrene nanoparticles) and free proteins showed no selective accumulation in the inflamed lungs when compared with naive lungs. The authors further show that complement proteins C3 and C5 opsonize NAPs, facilitating uptake by neutrophils. Nanoparticles lacking agglutination have little interaction with C3 and C5, suggesting that complement opsonization is the predominant route of neutrophil tropism of NAPs.

Myerson and colleagues further provide translational applications of NAPs for diagnosis of ALI/ARDS. Computerized tomography (CT) imaging detects pulmonary edema and is a standard diagnostic tool for ARDS. However, it poorly differentiates ALI/ARDS from non-inflammatory cardiogenic pulmonary edema (CPE), especially in the early stages of illnesses ${ }^{9}$. In single-photon emission computed tomography/computed tomography (SPECT/ CT) imaging, radiolabelled lysozyme-dextran nanogels, a prototype of NAPs, do not accumulate in the lungs of the CPE mouse model, but do accumulate in the lungs of the ALI/ARDS mouse model, confirming the specificity of NAPs to inflammation. In addition, the authors demonstrate that nanogels perfused ex vivo to inflamed human lungs are retained longer than ferritin nanocages, supporting the neutrophil tropism of NAPs in human lungs.

Importantly, liposomes, a type of non-protein nanoparticle, can be equipped with neutrophil tropism by conjugating agglutinated proteins, namely rat immunoglobulin $\mathrm{G}$ ( $\mathrm{IgG}$ ) functionalized with dibenzocyclooctyne (DBCO), a widely used alkyne for click chemistry. At a 20:1 molar ratio with respect to IgG, DBCO provides immunoliposomes with enough hydrophobicity to mimic NAPs, and neutrophil tropism. In addition, $\operatorname{DBCO}(20 \times)-\operatorname{IgG}$ liposomes, without any drug, appear to alleviate the course of ALI/ ARDS by decreasing neutrophil recruitment to the inflamed lungs and extravasation of proteins and leukocytes into the alveolar spaces. Although the mechanism underlying the therapeutic effects of $\operatorname{DBCO}(20 \times)$ IgG liposomes is unclear, the authors show that the liposomes reduce the levels of chemokines (CXCL2 and IL-6) in a dose-dependent manner.

A myriad of nanostructures are inspired from supramolecular assemblies in nature ${ }^{10}$; nonetheless, mechanistic understanding of how biological systems interact with supramolecular alignment remains limited. In this regard, the study by Myerson and 


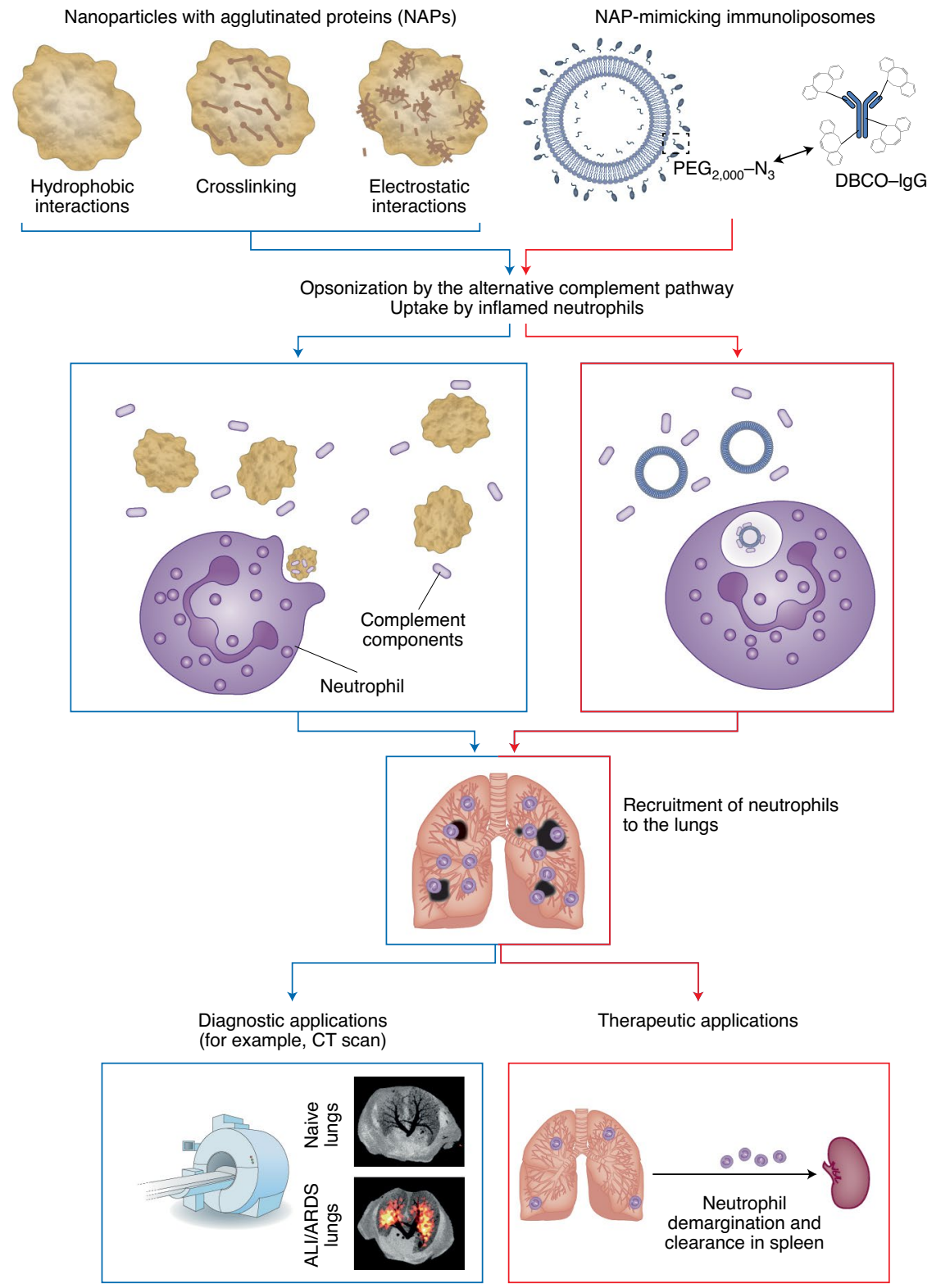

Fig. 1 | Uptake of neutrophils to inflamed lungs. NAPs assembled by hydrophobic interactions, crosslinking or electrostatic interactions (blue line) and DBCO(20x)--lgG liposomes (red line) are opsonized and activate the alternative complement pathway, resulting in selective uptake to neutrophils in an acute inflammation. Neutrophils carrying the nanoparticles are recruited to the inflamed lungs, and pulmonary localization of NAPs and DBCO(20x)-lgG liposomes enables diagnosis and treatment of ALI/ARDS, respectively. Figure adapted with permission from ref. ${ }^{8}$, Springer Nature Ltd. Created with BioRender.com.

colleagues is an exciting discovery that gives new insight to supramolecular assembly for medical applications. The interplay between agglutinated proteins on nanostructures and complement proteins raises the potential for novel approaches to immunotherapy. However, several important questions remain. First, it remains to be determined how $\mathrm{DBCO}(20 \times)$-IgG liposomes show anti-inflammatory effects whereas other NAPs do not show therapeutic benefits. Second, the diagnostic and/or therapeutic efficacy of NAPs and DBCO $(20 \times)$-IgG liposomes need thorough verification in various ALI/ARDS animal models. These nanoparticles may behave differentially in response to diverse chemokine profiles between species. In addition, the ALI/ARDS models used in this study are based on lipopolysaccharide-induced injury. It will be beneficial to investigate the efficacy of NAPs in other ALI/ARDS models induced by stimuli unrelated to lipopolysaccharide. Finally, to generalize the concept of protein agglutination, continued efforts to provide NAP-like properties in other nanostructures by crosslinking and electrostatic interactions are needed. Additional studies on the NAP biodistribution in mice lacking complement components and on its endocytosis could delineate the molecular mechanisms contributing to the neutrophil tropism. Nevertheless, this transformative discovery opens new avenues for nanoparticle-based diagnosis and treatment of inflammation.

Jeonghwan Kim (D)1 and Gaurav Sahay ${ }^{1,2,3 凶}$ ${ }^{1}$ Department of Pharmaceutical Sciences, College of Pharmacy, Oregon State University, Portland, OR, USA. ${ }^{2}$ Department of Biomedical Engineering, Oregon Health and Science University, Portland, OR, USA. ${ }^{3}$ Department of Ophthalmology, Casey Eye Institute, Oregon Health and Science University, Portland, OR, USA.

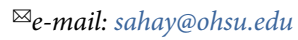

Published online: 18 November 2021

https://doi.org/10.1038/s41565-021-00981-6

References

1. Bellani, G. et al. JAMA 315, 788-800 (2016).

2. Tzotzos, S. J., Fischer, B., Fischer, H. \& Zeitlinger, M. Crit. Care 24, $516(2020)$.

3. Thompson, B. T., Chambers, R. C. \& Liu, K. D. N. Engl. J. Med. 377, 562-572 (2017)

4. Zemans, R. L. \& Matthay, M. A. Thorax 72, 1-3 (2017).

5. Vij, N., Min, T., Bodas, M., Gorde, A. \& Roy, I. Nanomedicine 12, 2415-2427 (2016)

6. Wang, Z., Li, J., Cho, J. \& Malik, A. B. Nat. Nanotechnol. 9 , 204-210 (2014).

7. Bisso, P. W., Gaglione, S., Guimarães, P. P. G., Mitchell, M. J. \& Langer, R. ACS Biomater. Sci. Eng. 4, 4255-4265 (2018).

8. Myerson, J. W. et al. Nat. Nanotechnol. https://doi.org/10.1038/ s41565-021-00997-y (2021).

9. Komiya, K., Akaba, T., Kozaki, Y., Kadota, J.-i. \& Rubin, B. K. Crit. Care 21, 228 (2017).

10. Kim, J., Narayana, A., Patel, S. \& Sahay, G. Theranostics 9 , 3191-3212 (2019).

Competing interests

The authors declare no competing interests. 